\title{
Editorial
}

https://doi.org/10.11646/megataxa.1.1.5

\section{Challenges for the future of taxonomy: talents, databases and knowledge growth}

\author{
CHARLES OLIVER COLEMAN ${ }^{1} \&$ ADRIANA E. RADULOVICI ${ }^{2}$ \\ ${ }^{I}$ Museum für Naturkunde Berlin, Leibniz Institute for Evolution and Biodiversity Science, Invalidenstraße 43, 10115 Berlin, Germany; \\ झ"oliver.coleman@mfn.berlin, (1) https://orcid.org/0000-0002-3051-7575 \\ ${ }^{2}$ Centre for Biodiversity Genomics, University of Guelph, 50 Stone Road E, Guelph (ON) N1H 2W1, Canada; \\ ”aradulov@uoguelph.ca, (1) https://orcid.org/0000-0002-2750-3850
}

The way taxonomy is done and communicated has changed over the last decades. When the first author started his career in biology some 35 years ago, most knowledge was organized on paper. Scientific books and papers, not present in the library, had to be obtained by a slow interlibrary loan. Photocopies with species information were arranged into taxonomic paper card files in order to overview thousands of taxa. For large taxa such a taxonomic card file took years to be built. We are all lucky that things have improved in the meantime and taxonomists can be much more productive today due to the digital revolution. Also, the taxonomic publication practice has changed. Tancoigne and Dubois (2013) showed, by analyzing data from the Zoological Record between 1980 and 2010, that the number of species per taxonomic publication slowly decreased in this time period, as well as the number of annually described new species or subspecies per author. They also found that the proportion of interdisciplinary publications increased between 1950 $(0.9 \%)$ and $2008(27.9 \%)$ and that monographs became less numerous. Sangster and Luksenburg (2014) showed similar trends in bird taxonomy comparing publications between 1935 and 2009. Interestingly the quality of the bird descriptions increased in this time interval for example by more detailed descriptions, inclusion of illustrations, maps and sonograms. It seems that taxonomy has become more detailed, interdisciplinary and thus more interesting over the years. And the publication process is faster today. Submitting manuscripts and plates as well as the reviewing process can be conveniently done using online portals. So, is everything fine and the future for taxonomy is bright?

Since the signature of the Convention on Biological Diversity during the 1992 Earth Summit in Rio de Janeiro the term "taxonomic impediment“ was recognized to sum up the problems of taxonomic knowledge gaps, the insufficient numbers of trained taxonomists and a lack of taxonomic infrastructure. Since then it has been highly controversial how significant the taxonomic impediment really is, but it seems to be a worldwide problem. For example, Venera-Pontón et al. (2020) mentioned a particular shortage of taxonomists and data from developing countries and countries in transition, especially in the fields of marine biodiversity. Additionally, Paknia et al. (2015) looked at the taxonomic infrastructure in megadiversedeveloping countriesandfound comparatively low numbers of biodiversity collections, natural history museums and herbaria. A factor to counter the taxonomic impediment is technological progress. Many taxonomic procedures are now facilitated by the use of computers, the internet, online species lists and molecular techniques. Now taxonomy is on the way to grow up to be a modern, integrative, hypothesis-driven and thrilling science.

In the following essay we want to shed some light on selected subjects of taxonomy that we think are worth looking at: the problems of the taxonomic workforce, the taxonomic knowledge growth based on the use of databases and web-portals and some suggestions for quality improvements in molecular databases for the benefit of the scientific community, conservation practitioners, regulators, policy-makers and citizen-scientists.

\section{Why is it so difficult to become a taxonomist?}

Becoming a taxonomist (in these paragraphs we think of a taxonomist as a classical species expert) requires some special talents, even perhaps a special type of brain in order to retain a complex classification of a group but also the minute differences that discriminate between species. For some, the interest in species starts at an early age, when parents and children explore the wonders of nature together, a very emotional and formative experience for both. Others have dedicated teachers at school or at university generating a spark in a young person to study species.

However, it seems that nowadays such a foundation in the interest of natural history is not laid so easily. For example, nature conservation organizations complain about diminishing numbers of new members and a high 
proportion of older ones. While species experts are desperately needed, for example for nature conservation, it seems that not enough experts are being educated (Frobel \& Schlumprecht 2014). The problem starts in schools, where today's curricula do not necessarily include natural history subjects, and continues in many universities, where taxonomy is not formally taught anymore and professors with taxonomic experience are missing. Moreover, the world today is fast-pacing and digital while the patience and observation skills required in taxonomy might be declining as traits of new generations. There are several studies reporting a sometimes only presumed decrease in taxonomists (e.g. Mora et al. 2011), others, however, do contradict this impression (Lohrmann et al. 2012). In recent years even politics has begun to express concern about the deteriorating taxonomic knowledge (House of Lords. Science and Technology Committee, 2008), especially with respect to some non-charismatic taxa (e.g. nematodes, fungi).

Be that as it may, it is not easy to become and remain a taxonomist and we think, it could be related to our modern science culture:

- Taxonomy is typically a long-term commitment. It takes a long time, usually many years, to become an expert on a group of species. And time is not easily available today. Young scientists are perhaps not willing to spend years on organizing taxonomic information in an analogous or digital way. If the taxonomist is a working group leader, then his/her students can participate in their preliminary work and there is a sense of knowledge tradition, e.g. during the Partnerships for Enhancing Expertise in Taxonomy (PEET) program of the National Science Foundation (NSF) (Rodman \& Cody 2003). However, if such an integrating figure retires, it leaves a large gap and student-based taxonomic projects may come to a stop.

- Science is project-based. After a particular project has finished, a very different topic might be taken up by the scientists. Research often follows current trends, which then change to new trends. So scientists must be very flexible today, and the least flexible is the taxonomist who is "married for life" to his/her taxonomic group and cannot easily switch fields or taxon because of the time already invested in the necessary groundwork.

- Taxonomy, at least classical taxonomy, is rather inexpensive, which seems to be good at first glance. But cheap taxonomic projects result in low overheads, which are not attractive for academic institutions. Would an institution hire a cheap taxonomist or prefer an applicant using modern, expensive methods, therefore attracting large grants? And modern methods also mean for a young scientist a good reputation and freedom to change to another field of science or the wider economy using the same techniques.
- Speaking of reputation, journals where taxonomists normally publish have notoriously low impact factors. Because the taxonomic community for most taxa is not large, the citation numbers amongst these few people are also low. To make matters worse, there is a practice unique in science not to cite species hypotheses properly, especially amongst users of taxonomy, e.g. ecologists, who in most cases do not cite species authors in their references. For example, Escherichia coli (Migula, 1895), a model organism used in many fields of modern science, has approximately 3.570.000 Google scholar hits, but the original author of the bacterium has only been mentioned in the text of 792 publications and fully cited in the references only 35 times (accessed $6^{\text {th }}$ January 2020).

For young scientists it would be strategically unwise to invest a lot of time to become a species expert and to bet on taxonomy for a future job opportunity. Many understand this and select other fields.

\section{How to ensure a high-quality knowledge growth in taxonomic portals and databases?}

Despite the often-mentioned complaint that taxonomy is in a kind of crisis, some say we live in the best of all times, because it has never been so easy to organize taxonomic knowledge so effectively. Indeed, today we can use internet-based species lists like the World Register of Marine Species (WoRMS) (http://www. marinespecies.org), databases for taxonomy like "DELTA", "Lucid" or "Scratchpads" (http://scratchpads. eu) and molecular databases such as GenBank and the Barcode of Life Data Systems ("BOLD") for molecular species identification (see below). We have modern digital inking methods, scanning electron microscopy (SEM) and stacking photography for high-quality illustration of species. Metadata from museum collections are being databased all over the world and are accessible via the Global Biodiversity Information Facility (GBIF) portal (https://www.gbif.org) making it easy to find material for answering specific taxonomic questions. Scientific literature has never been so easy to find and exchange as digital copies (pdfs, e.g. using researchgate. com or the Biodiversity Heritage Library, https://www. biodiversitylibrary.org).

But can these databases and collections enable a smooth transition of taxonomic knowledge from one generation of experts to the next? And are they curated well enough to be useful, and have workflows implemented so that mistakes can be ironed out easily?

- What taxa do we know? There is a large number of such databases with species lists, some curated by a single person, others by large teams. Some of these databases mirror or aggregate data amongst each other. ZooBank 
(http://zoobank.org/) is the official registry of zoological nomenclature and it is automatically filled with data during the taxonomic publication process.

From the large list of databases available online, we are highlighting only two examples of good practice in terms of data management.

"World Register of Marine species", WoRMS (http:// www.marinespecies.org). At the moment there are 289 editors, supervising taxa they are responsible for and very quickly updating the species list or changes to the classification once new information becomes available. Some of the higher ranks have separate sub-databases, e.g. "World of Copepods" and the "World Amphipoda database", the latter having three coordinating editors and 31 associate editors. These sub-databases directly feed into the main database of WoRMS.

The "World Spider Catalog" (https://wsc.nmbe.ch) (Nentwig et al. 2015). This is not only a species list on spiders, but also offers access to 15.000 taxonomic papers as pdfs for members. Similar to WoRMS, there is a team of subject editors ensuring the quality of the database.

\section{- How to identify taxa with software and use such} tools to write taxonomic papers? There are databases for taxonomic characters that dramatically speed up writing species descriptions, creating keys and interactive identification tools. "DELTA", Descriptive Language for Taxonomy (Dallwitz 1980) (https://www.delta-intkey. com; https://downloads.ala.org.au/p/Open\%20DELTA) is an example of a free of charge, respectively open-source, software package. "Lucid" (https://www.lucidcentral. org) concentrates on creating keys on the web. It is a commercial product and thus not open-source. The future of this product and the databases created with it is therefore questionable. But also interactive keys created with free software such as "DELTA" may be taken off the internet when the promotor retires (e.g. Dr. Jim Lowry, who created and was running http://crustacea.net, a website with interactive keys and information on many crustacean taxa). Isolated projects, running only for a limited time, do not contribute much to the growth and maintenance of taxonomic knowledge. Another problem of such isolated applications is that they are run by individuals only and are not designed for larger cooperative work on the same database at the same time. However, this disadvantage could easily be overcome as the DELTA software now is open-source and will certainly be further developed in the future. Also, the future of projects made with "Scratchpads" (http://scratchpads.eu), a content management system primarily designed for taxonomic contents, remains unclear when developed by a single person. It may stop being updated when the author retires or switches jobs. In such cases there is not much of a tradition of knowledge.
- Where is the reference material stored? If museum collection metadata are captured and published on the web, sometimes thanks to the internet publication, the material starts to "exist" for the users. For type material in a collection, the (original) name of a species is clear and relatively stable. For the non-types, however, we would have to expect a certain misidentification rate (e.g. more than $50 \%$ of tropical plant specimens were incorrectly named in herbaria, according to a case study on some taxa by Goodwin et al. 2015) and constant work on these specimens is necessary. For collections that are regularly worked on, the quality of identification and metadata should increase with use over the years. When collections are not databased and are not being used, the status often remains frozen. Also, the organization of collection departments of museums has a strong influence on the quality of a collection. If collections are not actively worked on by teams of scientists and technicians, the collection may become neglected and then decline from a well-organized system to an unorderly aggregation. However, in many institutions such cooperation does not exist anymore: scientist do their science and technicians or collection managers (sometimes with limited taxonomic expertise) feel left alone with the collection - then the quality of a collection may suffer. In some museums it is becoming difficult to get types on loan (e.g. Naturalis, Leiden). In other cases, only a few specimens are deposited in the collections and a large number stay in the private working collections of the taxonomists with an unclear fate for the future.

\section{- What is needed?}

- If projects are financially supported for only a limited time, the future of the database should be planned ahead to ensure the sustainability of the database.

- Individual operators of databases should find successors for their projects, best connected to a larger institution.

- Larger portals should be run by large institutions with a sound financial basis and long-term accessibility must be guaranteed.

- Databases on the web should have interfaces for cooperation and improvements of the contents.

- Finally, museums should have teams of scientists and technicians working and developing the collections and their metadata together

\section{What are the challenges of molecular databases for species identification?}

The science fiction of future species identification has already been outlined: a handheld device is pressed on a specimen and the species name appears on the display. Whereas the scanner is until now only a dream (although progress in technology keeps advancing in this direction), the underlying databases for DNA-based 
species identification are already a reality. GenBank, the largest database of genetic sequences, has been populated with data by scientists during the publication process of their peer-reviewed articles (https://www.ncbi. nlm.nih.gov/genbank/). Although a large database with millions of sequences that can be queried by any user free of charge, GenBank has limited metadata that can be uploaded together with sequence data. Moreover, in case any error has slipped (most often taxonomic errors due to misidentification), only one of the original authors can correct it which brings difficulties in cases where authors have retired, changed addresses or jobs.

For the rest of this section, we will focus on the Barcode of Life Data System (BOLD; http://www.boldsystems.org; Ratnasingham and Hebert, 2007), a database dedicated to DNA barcodes for species identification. As opposed to GenBank, BOLD is only focusing on multi-cellular organisms and it currently contains 7.7 million barcodes of 216.000 animal, 69.000 plant and 22.000 fungal and other species but also including a certain number of sequences without species-level identification (accessed $12^{\text {th }}$ January 2020). These data were uploaded directly by BOLD users or mined from GenBank by the BOLD team. A large amount of the data directly uploaded to BOLD were generated through the efforts of the International Barcode of Life Consortium (https://ibol.org/). Currently, iBOL has 32 member states but we are presenting only one example of good practice here.

The German branch of the iBOL Consortium has been very active since its inception as the German Barcode of Life (GBOL). This national network has strict quality requirements of DNA barcoding data and metadata when building reference libraries. Only sequences of specimens identified by acknowledged and registered species experts are accepted and these reference specimens (i.e., voucher specimens) of sequenced material are photographed and then deposited in GBOL partner museums or university collections. Up to now (accessed $6^{\text {th }}$ January 2020), 17.624 animal species (203.859 specimens), 1.303 plant species (3.402 specimens) and 9 fungi species (14 specimens) were listed on the GBOL website (https:// www.bolgermany.de).

However, not all BOLD users follow the same quality standards as GBOL. As a consequence, BOLD (as well as GenBank and other molecular databases) contains a certain amount of data that was not properly validated and might affect correct identification based solely on DNA barcodes. The issue of incorrect taxonomic identification is not exclusive to molecular databases as it frequently occurs also in museum/herbaria collections (as mentioned above). Being a database as well as a workbench for data analysis, BOLD has a set of recommendations for high-quality data and metadata (e.g., name of taxonomist identifying the specimen, name of institution storing the specimen, image of the specimen, biological details of specimen, collection data, DNA sequence and electropherogram etc.), but the ultimate responsibility of following these recommendations lies with the user.

A few years ago, we performed a test of data quality in BOLD with regards to public amphipod data (Radulovici \& Coleman 2017). We already suspected that errors might have crept into BOLD based on a research visit of one of us (C. O. Coleman) to a well-known national collection. The visit included a revision of a collection of amphipods which had been identified and barcoded previously. From every tube containing multiple specimens of supposedly, the same species, an amphipod was selected and processed through standard barcoding protocols and data uploaded to BOLD. In many tubes, however, the amphipods belonged to several species, sometimes even from different families. This finding, together with the difficulty of tracing the actual specimens that were barcoded in order to verify their identification, led us to suspect a potential erroneous input into BOLD. Checking the respective amphipod data in BOLD, we found that it was not meeting the desired quality standards. In many cases there were no pictures associated with the sequenced specimens and, if present, the pictures were not able to help resolve problematic cases. Many pictures were small or showed only the habitus of the amphipod, but no essential details of appendages or mouthparts required to see taxonomically relevant characters. Due to the morphological complexity of these animals, such photos are not suitable to check the validity of the identification. Most amphipods cannot be identified to species level only by their habitus. In some cases, the same picture has been uploaded for multiple specimens which, when processed, generated very distant DNA sequences, indicating multiple species. In other cases, pictures clearly showing different species were uploaded for specimens of the same species. One picture showed a completely different family in contrast to the one mentioned in BOLD. In other BOLD projects, pictures showed only meaningless body fragments left after tissue extraction or rough dissection during the identification. In a nutshell, most amphipod pictures in BOLD were not helpful for documenting the species (Fig. 1).

To help with the widespread taxonomic issues, BOLD implemented an algorithm which clusters animal barcodes in cohesive groups with unique identifiers called Barcode Index Numbers (BINs; Ratnasingham \& Hebert 2013). These BINs were shown to largely correspond to Linnean species in multiple taxa, and therefore are used as proxies for species. While the inventory of barcoded animal species includes 216.000 species, the number of BINs is three time higher $\left(659.000\right.$ BINs on $12^{\text {th }}$ January 2020) indicating the presence of potential cryptic species but also of many unidentified species present in the database. Due to available tools based on BINs (e.g., BIN 
discordance report), misidentifications can be easily found when one particular BIN includes multiple species names. The BOLD team is routinely performing data curation and investigating cases of discordant BINs, however the process is not automated to date. Manual curation is employed and suspect records are flagged and excluded from the dataset available to users when querying their unknown sequences. Since BINs are generated every month based on new data added to BOLD while the curation is manual and performed periodically, it follows that at any given moment erroneous data might exist in BOLD. A similar test to the one in 2017 was performed again in 2019 and found that $8 \%$ of the public amphipod BINs had discordance (multiple taxa in one BIN) (Radulovici 2019). For rare species, however, where a $\mathrm{BIN}$ is based on only one sequence or no BIN is available (i.e., short DNA sequences are not generating a BIN), a mistake in identification might remain unrecognized for a long time, if recognized at all. Overall, however, misidentifications in the "molecular system" are much easier and faster to detect and correct compared to the "traditional taxonomic" system.

\section{Consequences of factually flawed metadata:}

BOLD has been established as part of the DNA-based identification system for the multi-cellular life on Earth. Through this approach, the inventory of life can be performed in a faster, standardized, simplified and costeffective way and in doing so, new species are bound to be found. With new technological advances, it is easier to produce massive amounts of DNA data than ever before. Bulk samples or environmental samples can now be routinely processed as part of biodiversity assessments and monitoring. However, the success of such approaches in reaching a final species list is based largely on the reliability and completeness of the database available to users when performing their queries for their new data. Therefore, it is essential that each reference sequence in BOLD (and other molecular databases for that matter) is connected to the correct taxon. And if misidentified specimens are connected to sequences in the database and go unnoticed, these mistakes may linger for a very long time affecting to a certain degree all the studies that relied exclusively on molecular databases in the meantime.

Although occurring less frequently, what happens if there is only a single specimen and this is destroyed during tissue extraction or completely used due to small size? This might happen if the researchers are not following special protocols for voucher recovery (e.g. cuticle of a specimen saved after DNA extraction, which even worked for minute kinorhynchs, Yamasaki 2015) or are not careful enough during laboratory protocols. These potential cases are especially critical if the species is rare. Then the link between organism and sequence can no longer be tested.

\section{Suggestions to address these problems:}

BOLD is an online platform used by thousands of users around the world. As such, it relies heavily on users being already trained in best practices for DNA barcoding which might not always be the case as we have seen in amphipods. At the moment, records that are suspected to be contamination or misidentifications are flagged and would not hinder the identification tool implemented in BOLD. However, this action does not necessarily 'fix' the problem, especially for records mined from GenBank which lack metadata.

- A possible solution would be the implementation of a system of volunteer editors (taxonomists with expertise for different taxa), similar to the established system in WoRMS (see above) to keep track and curate data uploaded to BOLD.

- Furthermore, we specify below the metadata that would greatly improve the quality of BOLD if followed by all users. Although all these fields are currently available in BOLD, few users are filling out all this information as they upload DNA sequences.

- Metadata should include, the following:

- 1) Extensive illustrations, with photos and/or drawings of all relevant characters for identification, for every sequenced specimen of the reference library (or at least one specimen from each BIN);

2) Exact locality of collection with GPS coordinates;

3) Accession number of collection where reference material is stored;

4) Name of the person who identified each specimen and the date;

- 5) Pdf of the publication once the sequence has been published;

6) BOLD has a system of tags and comments that is overall underutilized. Each record can receive comments or tags allowing data to be curated faster;

- 7) The placement of reference material in museums, similar to the procedure of morphological types, should be obligatory.

While BOLD could implement some of these recommendations as mandatory fields for the benefit of the barcoding community, this action could be seen as a barrier to uploading data to BOLD by the same barcoding community. We believe that the users bear the responsibility to generate, validate and curate their data even, and especially, after publication. The barcoding community can learn a great deal from taxonomists who would revisit museum collections again and again over time to improve specimen identification to the benefit of the scientific community at large. As a community, we need to establish and follow a code of best practices to improve the BOLD system in order to ensure its longterm usefulness. 


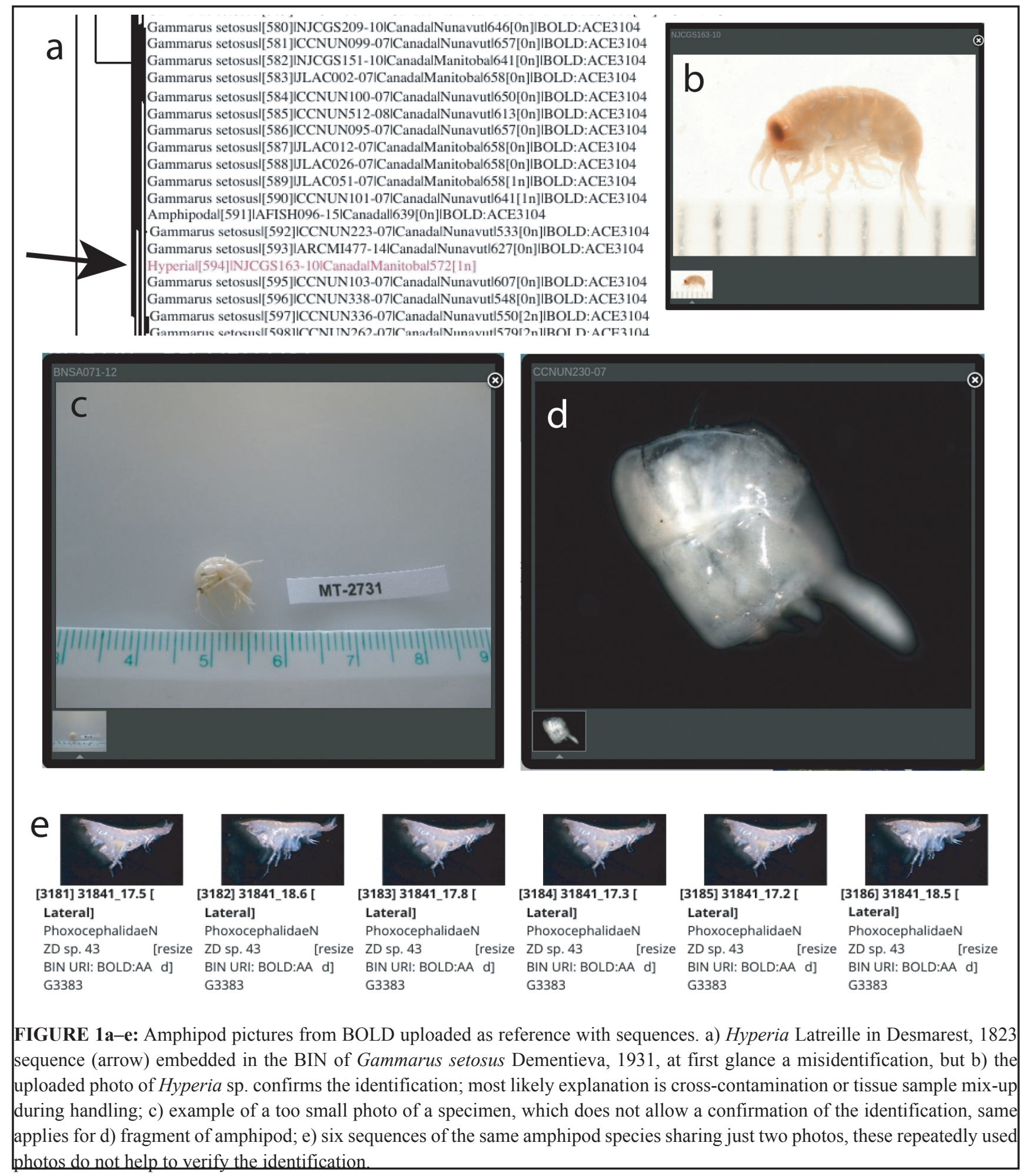

\section{Concluding remarks}

Taxonomy is a complex but fascinating field. Identifying organisms can take anywhere from minutes to hours or days, and it can be done by eye or by using ever more advanced microscopes and dissecting tools. Only the person who struggled through endless dichotomous keys and uncertainties can understand the satisfaction of arriving at the end, at a species name for the organism at hand. Whether based on morphological characters or a string of nucleotides, species identification is important. The entire field of biodiversity conservation has species identification as starting point. And since biodiversity is in crisis, there is a need for trained experts in species identification as never before. No molecular database is complete; we are not even close to have barcoded all the species inventoried to date, let alone the many more that are estimated. The multitude of cryptic species discovered 
through molecular methods needs taxonomists to classify and describe them. Only by working across disciplines and breaking stereotypes, will we be able to advance the taxonomic knowledge and understanding of the natural world. This essay is presenting our view on the state of the taxonomy today. We hope that it has not deterred any student or early career researcher or even citizen-scientist from considering taxonomy as a work field, by itself or as an add-on to other fields of research.

After all, taxonomy is becoming a modern, integrative, hypothesis-driven and thrilling science like never before.

\section{Acknowledgements}

We would like to thank Dr. Birger Neuhaus and Dr. Jason Dunlop (both Museum für Naturkunde Berlin) for their suggestions and improvements of the manuscript.

\section{References}

Dallwitz, M.J. (1980) A general system for coding taxonomic descriptions. Taxon, 29, 41-46. Available from: http://deltaintkey.com/www/dallwitz-1980.htm (Accessed 17 Jan. 2020)

Dementieva, T. (1931) On the variability of the Amphipoda of the northern seas. Akademiia Nauk SSSR, Trudy Okeanolografii Instituta, 1, 65-82.

Frobel, K. \& Schlumprecht, H. (2014) Erosion der Artenkenner. BUND Naturschutz in Bayern e.V., Nürnberg, 92 pp.

Goodwin, Z.A., Harris, D.J., Filer, D., Wood, R.I. \& Scotland R.W. (2015) Widespread mistaken identity in tropical plant collections. Current Biology, 25(22), R1057-R1069. https://doi.org/10.1016/j.cub.2015.10.002

Latreille, P.A. (1823) Malacostracés. In: Desmarest, A.G. (Ed.) Dictionnaire des sciences naturelles, dans lequel on traite méthodiquement des différens êtres de la nature, considérés soit en eux-mêmes, d'après l'état actuel de nos connoissances, soit relativement à l'utilité qu'en peuvent retirer la médecine, l'agriculture, le commerce et les artes. Suivi d'une biographie des plus célèbres naturalistes. Strasbourg, Paris, Vol. 28, pp. $138-425$.

Lohrmann, V., Vohland, K., Ohl, M. \& Häuser, C. (2012) Taxonomische Forschung in Deutschland. Netzwerk-Forum zur Biodiversitätsforschung Deutschland (NeFo), pp. 1-122.

Migula, W. (1895) Bacteriaceae (Stäbchenbacterien) In: Engler, A. \& Prantl, N. (Eds) Die Natürlichen Pflanzenfamilien. Teil I, Abteilung Ia, W. Engelmann, Leipzig, pp. 20-30.
Mora, C., Tittensor, D.P., Adl, S, Simpson, A.G.B. \& Worm, B. (2011) How many species are there on earth and in the ocean? PLoS Biology, 9, 1-8. https://doi.org/10.1371/journal.pbio.1001127

Nentwig, W., Gloor, D. \& Kropf, C. (2015) Taxonomic database: Spider taxonomists catch data on web. Nature, 528, 479. https://doi.org/10.1038/528479a

Paknia, O., Rajai Sh., H. \& Koch, A. (2015) Lack of well-maintained natural history collections and taxonomists in megadiverse developing countries hampers global biodiversity exploration. Organisms Diversity \& Evolution, 15, 619-629. https://doi.org/10.1007/s13127-015-0202-1

Radulovici, A.E. \& Coleman, C.O. (2017) Reconciling large molecular datasets, bioinformatics and taxonomy: prospects for Amphipoda. Biodiversity Journal, 8(2), 633-634.

Radulovici, A.E. (2019) What public data can tell us about current practices in DNA barcoding amphipods. $18^{\text {th }}$ International Colloquium on Amphipoda, Dijon, France, 26-30 August 2019, Book of Abstracts, pp. 66.

Ratnasingham, S. \& Hebert, P.D.N. (2007) BOLD: The Barcode of Life Data System (www.barcodinglife.org). Molecular Ecology Notes, 7, 355-364. https://doi.org/10.1111/j.1471-8286.2007.01678.x

Ratnasingham, S. \& Hebert, P.D.N. (2013) A DNA-Based Registry for All Animal Species: The Barcode Index Number (BIN) System. PLoS ONE, 8, 8, e66213. https://doi.org/10.1371/journal.pone.0066213

Rodman, J.E. \& Cody, J.H. (2003) The taxonomic impediment overcome: NSF's Partnerships for Enhancing Expertise in Taxonomy (PEET) as a model. Systematic Biology, 52, 428435. https://doi.org/10.1080/10635150390197055

Sangster, G. \& Luksenburg, J.A. (2015) Declining rates of species described per taxonomists: slowdown of progress or a sideeffect of improved quality in taxonomy? Systematic Biology, 64, 144-151. https://doi.org/10.1093/sysbio/syu069

Tancoigne, E. \& Dubois, A. (2013) Taxonomy: no decline, but inertia. Cladistics, 29, 1-4. https://doi.org/10.1111/cla.12019

Venera-Pontón, D.E., Driskell, A.C., De Grave, S., Felder, D.L., Scioli, J.A. \& Collin, R. (2020) Documenting decapod biodiversity in the Caribbean from DNA barcodes generated during field training in taxonomy. Biodiversity Data Journal, 8, e47333. https://doi.org/10.3897/BDJ.8.e47333

Yamasaki, H. (2015) Two new species of Dracoderes (Kinorhyncha: Dracoderidae) from the Ryukyu Islands, Japan, with a molecular phylogeny of the genus. Zootaxa, 3980 (3), 359378.

https://doi.org/10.11646/zootaxa.3980.3.2 